\title{
Inevitable failures in geometric arrangement during bone remodeling determine irreversible bone loss: a study by Monte Carlo simulations
}

\author{
Roosevelt A. da Silva ${ }^{1}$, Heberton S.C. de Souza², Ismael V.L. Costa ${ }^{1}$ \\ and Henrique A. Fernandes ${ }^{1}$
}

Manuscript received on October 20, 2010 / accepted on December 9, 2011

\begin{abstract}
Bone is a dynamic tissue that is constantly being remodeled by the action of osteoclasts $(\mathrm{OC})$ and osteoblasts (OB), which are responsible for bone resorption and formation, respectively. The mechanism through which these cells help the bone tissue remain healthy is still unclear. Great efforts have been made toward the elucidation of bone loss, especially in the case of menopausal women, in order to lessen the effects of osteoporosis. It is known that protein and hormonal alterations may lead to an imbalance in the activities of $\mathrm{OC}$ and $\mathrm{OB}$, thereby favoring the onset of bone disease. Other causes that are not associated with this imbalance are currently under investigation. We have carried out computer simulations in order to identify topological arrangements that could result in natural bone loss during the remodeling process. On the basis of a $2 \mathrm{D}$ model, which treats the $\mathrm{OC}$ and $\mathrm{OB}$ cells explicitly, bone remodeling was guided by an $\mathrm{OB}$ activation function of the $1 / r^{4}$ type ( $r$ = distance to the site resorbed by $\mathrm{OC}$ ), whose value determines bone formation when a predefined Activation Threshold (AT) is exceeded. We have verified the existence of a critical AT that can significantly affect bone mass balance, thus producing failures in the geometric arrangement of the trabecula. These failures become inevitable and irreversible with age.
\end{abstract}

Keywords: Monte Carlo simulations, bone remodeling, osteoporosis.

\section{INTRODUCTION}

Bone remodeling is a dynamic process carried out by osteoclast (OC) and osteoblast (OB) cells, which work together as a basic multicellular unit (BMU). In this process, old bone is removed from the skeleton (bone resorption) and new bone is added to it (bone formation). An imbalance in the relationship between the activities of $O C$ and $O B$ leads to many metabolic bone diseases. The interactions between $\mathrm{OB}$ and $\mathrm{OC}$, known as coupling, guarantee a proper balance between bone gain and loss [1].

Osteoporosis (OP) is a common disease of the skeletal system, characterized by reduction in the bone mass and degeneration of its microstructure. From a clinical viewpoint, this condition promotes high susceptibility to systemic fractures [2]. Osteoporosis has become a major health concern since there has been a sharp increase in the prevalence of $\mathrm{OP}$ among the elderly over the last decades [3].

\footnotetext{
Correspondence to: Roosevelt A. da Silva

${ }^{1}$ Universidade Federal de Goiás, Jataí, GO, Brazil.

2 Instituto de Pesquisa e Desenvolvimento, Universidade do Vale do Paraíba, São José dos Campos, SP, Brazil

E-mail: rooseveltfisicaufg@gmail.com
} 
From a mechanical perspective, fractures in osteoporotic individuals result from alterations in the bone structure, which make it less resistant to mechanical stress. This may be due to either a decrease in bone mass or the daily load demand applied on the same bone region $[2,4,5]$. Such bone mass loss is a consequence of the imbalance in the dynamics of the BMU, which may lead to negative bone formation in relation to bone resorption.

This BMU imbalance may be caused by protein and hormonal changes, as well as other biochemical and physical factors that influence the processes of cell activation and differentiation. An imbalance in the production of cytokines affects the activities of $\mathrm{OB}$ and $\mathrm{OC}$, thereby leading to common bone diseases. Osteoporosis is a very common example of a disease that may be caused by excess of the osteoprotegerin cytokine [16].

Bone inactivity or low mechanical stress may also affect osteoclast differentiation [17]. The bone structure generates mechanical signals at the cellular level upon mechanical stress, but their exact nature remains unknown $[18,19]$. The regulation of bone remodeling is complex, and it involves many factors that can influence both bone formation and resorption [20].

During menopause, bone loss is often associated with the aging process responsible for decreased hormonal activity, as in the case of estrogen [22]. Lack of estrogen may raise the frequency of osteoclast activation and reduce its apoptosis [23]. However, it has recently been verified that rapid bone loss in osteoporosis can also be explained by mechanobiological processes [24]. It has been found that an increase in the resorption cavity leads to bone loss by continuous resorption of damaged tissue. In this same context, it has been shown that the rate of the local growth of tissue formed by osteoblasts is strongly influenced by the geometrical features of channels in an artificial tridimensional matrix [25]. The geometric arrangement of the local environment can be a critical factor in determining cell behavior and, consequently, the bone growth patterns.

Computer simulation model two-dimensional has allowed to explain adaptations observed in trabecular bone in a conceptual sense [26]. In this study we have employed a computer simulation of bone remodeling in order to obtain a more detailed description of this process. We have also investigated the possible effects of geometric arrangement failures on bone mass loss, particularly in menopausal women and in the elderly.

We introduce a 2D model where osteoblast and osteoclast cells are considered explicitly, so that fundamental aspects of the dynamics of these cells can be captured. Because osteoblast and osteoclast cells provide model parameters, such as their activi- ties, that can be varied independently through the osteoblast and osteoclast cell rates, we expect to distinguish the differences between the effects of such rates from those effectively generated from geometric arrangement during the remodeling process.

\section{MATERIALS AND METHODS}

\subsection{System representation and initial configuration}

A 2D-lattice consisting of $n$ sites $(50 \times 50)$ was employed to represent the system. The sites were defined as being unoccupied or occupied by Trabecular Bone (TB), OB cells, or OC cells.

$T B$ was generated in a regular arrangement in the initial system configuration, as in the procedure employed by Huiskes [26]. Figure 1 depicts the initial TB state, where each trabecula has a width equal to $2 s$ ( $s=$ site) in the initial configuration, with a $4 s$ distance between them.

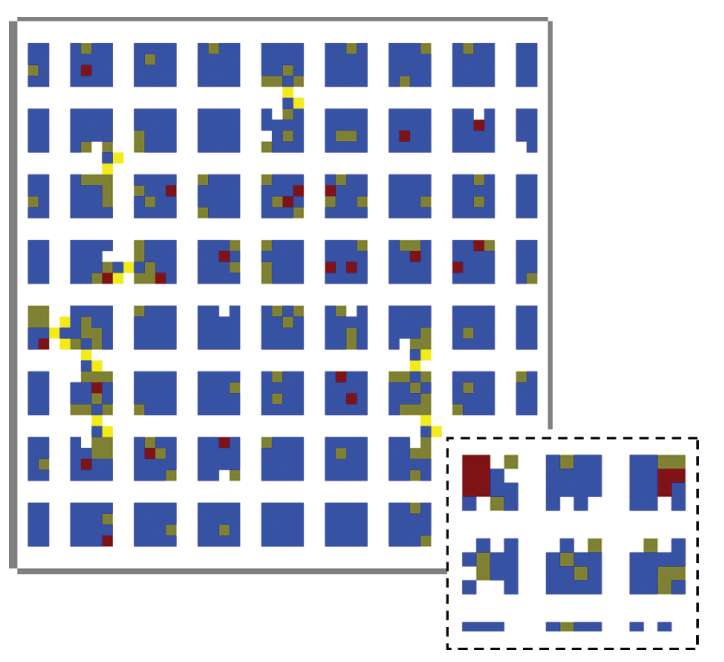

Figure 1 - Initial configuration: white sites correspond to the trabecular bone, where remodeling occurs; blue gaps are interstitial spaces; red and green cells represent osteoclasts and osteoblasts, respectively. The insert, enlarged from a part of the system, corresponds to the simulation that considers $\varepsilon_{O C-O C}=2.5 u$, where the formation of a cluster of $O \mathrm{C}$ cells mimicking multinucleated $\mathrm{OC}$ cells can be observed.

Initially, the $\mathrm{OB}$ and $\mathrm{OC}$ cells were randomly placed in the system. The densities of these cells were previously defined as a function of the ratio between the sites occupied by $\mathrm{OB}$ and $\mathrm{OC}$ and the total number of sites present in the lattice. In the initial configuration, no OC cell was initiated in its active state.

\subsection{Resorption and deposition}

The total energy involved in a particular system configuration is defined as,

$$
E_{\text {total }}=E_{\text {local }}+E_{S}
$$


where $E_{\text {local }}$ is the local contact energy between neighbor cells and $E_{S}$ is the surface energy generated from TB removal (which will be further described in Eq. 4). The term $E_{\text {local }}$ measures the amount of energy between the neighbor cells by means of the parameter $\varepsilon$; that is,

$$
E_{\text {local }}=\sum_{i, j} \varepsilon_{i j} \delta_{i j}
$$

In this equation, the $\delta_{i j}$ matrix identifies the sites that are closest to the network. Therefore, when an $i^{\text {th }}$ site is the closest neighbor to the $j^{\text {th }}$ site, it is said that $\delta_{i j}=1$; otherwise $\delta_{i j}=0 . \varepsilon_{i j}$ is the energy involved in the interaction between the $i$ and $j$ sites, whose values can be obtained from the type of cell present in $i$ and $j$, and includes interactions between the OB-OB, OB-OC, OB-TB, OC-OC, and OC-TB cells.

The values of $\varepsilon_{i j}$ in Eq. 2 are ascribed in such a way that the interactions are able to lead the system to typical states that can also be observed experimentally. For instance, a magnitude of $-2.5 u$ for $\varepsilon_{O C-O C}(u=$ arbitrary energy unit) and $\beta=1$ results in a stable contact between $O C$ cells, mimicking the conditions observed in the case of cells generated from the multinucleation of mononuclear osteoclasts (giant cells). On the other hand, a magnitude of $\varepsilon_{O C-T B}=-0.2 u$ for may lead to a moderate stability for the contact between $\mathrm{OC}$ and $\mathrm{TB}$, which is enough to favor a shift of $\mathrm{OC}$ beneath the trabecula at the same time it can freely move from one site to another.

\subsection{Osteoclast and osteoblast activity}

An OC cell located in a certain site $i$ can only absorb TB from a neighbor site $j$ when it is active. When $O C$ is selected for displacement from one site to another, a new activation state will always be conferred to it by means of the activation probability $\Omega$, given by the equation

$$
\Omega_{i j}=\left\{\begin{array}{llll}
1 & \text { if } & \text { rand } & \leq p_{0} \\
0 & \text { if } & \text { rand } & >p_{0}
\end{array}\right\},
$$

where rand is a number between 0 and 1 that is randomly obtained, and $p_{0}$ is the expected TB activation probability in the absence of stress. In this way, when $\Omega_{i j}=1$, the $0 \mathrm{C}$ cell in $i$ is active and capable of removing TB from the site $j$ (Fig. 2a). When $i$ has more than one neighbor (neighbors $j, k, l$, and $m$ ), the choice of which neighbor will be removed shall be random.
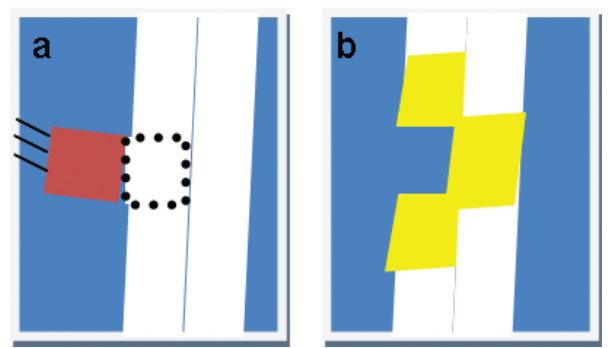

Figure 2 - Bone resorption. a) Osteoclast cell (red) approaching the surface of the trabecula for absorption (dotted line denotes the $i^{\text {th }}$ site involved in the removal). b) Sites next to $i$ (yellow) activated after $i$ removal.

When a TB cell is removed from a site by $\mathrm{OC}$, all the neighboring TB sites are perturbed (Fig. 2b), and a surface energy responsible for the recruitment of $\mathrm{OB}$ cells comes into effect as

$$
E_{S}=\sum_{i, j} \frac{\rho}{d_{i j}^{4}}
$$

where $d_{i j}$ is the distance between the $i^{\text {th }}$ osteoblast and the $j^{\text {th }}$ site activated by osteoclasts (yellow sites in Fig. 2b) and $\rho$ is a positive constant.

Although the perturbed sites (PS) by osteoclasts can generate activated neighbors, thus resulting in the rapid approach of $O B$ cells, deposition will only occur when the value of $E_{S}$ (Eq. 4) is above the pre-defined Activation Threshold (AT). If this condition is satisfied, $\mathrm{OB}$ will be able to deposit bone in one of its neighboring sites if the following requirements are also met:

I. $E_{S}>\mathrm{AT}$, and

II. when its neighbor is next to TB (see Fig. 3, cases I and II).

If the aforementioned conditions are satisfied, this site will become a potential TB, here defined as deposition site (DS). If other DSs are present in the surroundings of $\mathrm{OB}$, the choice of deposition site is random.

Still, if OB approaches DS, and if the latter is occupied by another OB (Fig. 3, case II), DS becomes a TB site independent of the presence of $\mathrm{OB}$. This corresponds to the conversion of an osteoblast into an osteocyte when $\mathrm{OB}$ is captured by the bone matrix. Whenever an $\mathrm{OB}$ cell is converted into TB as pointed above, a new $\mathrm{OB}$ is randomly inserted into a site of the system.

Finally, when an $\mathrm{OB}$ cell close to DS has $E_{S}<\mathrm{AT}$, TB deposition does not take place, and an accumulation of $\mathrm{OB}$ cells can occur in the surroundings of DS (Fig. 3, case III). Following osteoblast deposition, the remodeling cycle in that site is halted, and remodeling can only happen again when activated OC cells establish a new contact with this site. 
A

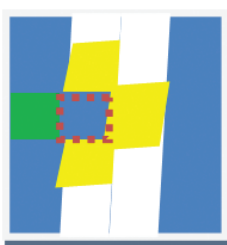

Case I

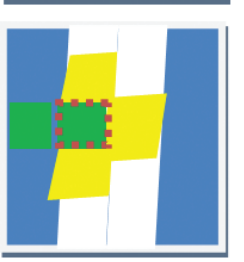

Case II
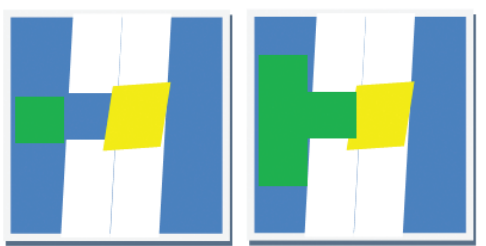

Figure 3 - Osteoblast action - Case I: A) OB (green) approach to DS surrounded by three active sites (yellow) and B) TB (white, dotted) just deposited by OB. Case II: A) OB approach to DS containing another OB cell and B) replacement of $O B$ with TB in DS. Case III: A) OB approach to DS surrounded by 1 active site (yellow), the $E_{S}$ energy of the approaching $\mathrm{OB}$ is lower than $\mathrm{AT}$, and B) accumulation of $\mathrm{OB}$ cells in the surroundings of DS due to $E_{S}$.

When a TB cell is removed from an $i^{\text {th }}$ site of the network by $\mathrm{OC}$, it is also necessary to assess the presence of bone fragments that are not associated with the network. This can be accomplished by verifying whether the TB neighbors of the site $i$ can make contact with the system borders by means of consecutive sites of the TB type. This verification is carried out upon each removal with the aid of a recursive function that is able to identify, from $i$, whether there is percolation via sites of the TB type only. If this is not the case, the fragment is eliminated from the trabecular lattice, and the corresponding energy is subtracted from the total energy of the system (Fig. 4).
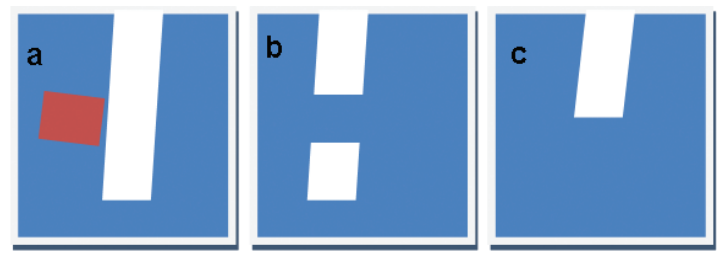

Figure 4 - Schematic representation of TB elimination. a) Osteoclast (red) acting on the bone surface (white); b) bone after removal of one TB site; $c$ ) removal of a TB fragment that is not in contact with the network borders.

\subsubsection{Movements of $\mathrm{OB}$ and $\mathrm{OC}$ cells}

A new system configuration is always attempted when a certain $O B$ or $\mathrm{OC}$ cell is moved. Initially, an $i j$ site containing either $\mathrm{OB}$ or
OC is randomly selected. Next, its position is modified to one of its first nviz neighbors or it can remain in its own site. In other words, the movement can occur in the $[i \pm n v i z, j \pm n v i z]$ range.

The transition of a cell from the $X$ state to a $Y$ state follows the metropolis criteria [27], whose probability $p(X \rightarrow Y)$ is given by

$$
p(X \rightarrow Y)=\min [1, \exp (-\beta \Delta E)],
$$

where $\beta$ is a constant related to the system fluctuation and $\Delta E$ is the difference between the energies of the configurations $Y$ and $X$; therefore,

$$
\Delta E=E(Y)-E(X),
$$

and $E$ can be obtained by Equation 1 .

\subsection{Initial parameters}

A total of $1.2 \times 10^{9}$ random configurations are produced for each simulation accomplished consecutively. A Monte Carlo Step (MCS) corresponds to 120 attempts to move or alter the state of a site/neighborhood. Therefore, each simulation comprises $10^{7}$ MCSs.

The osteoclast and osteoblast densities were defined as 0.05 and 0.01 , respectively. Although this difference results in five times more cells in the case of $\mathrm{OC}$, osteoclast nucleation is driven by the contact energy $\varepsilon$ (Eq. 2), so that $\mathrm{OC}$ cell clusters corresponding to multinucleate osteoclasts (MO) are formed. In this way, the number of $\mathrm{MO}$ is comparable to the total number of OB cells.

An nviz value of 10 was chosen so that, according to the $2 \mathrm{D}$ model defined here, the $\mathrm{OB}$ and $\mathrm{OC}$ cells would be able to move from one trabecula to another with no ergodicity problems [28]. At the same time, nviz was minimized so as to maintain the locality features of the system, somehow enabling one to capture the cell dynamics. In a 3D model, nviz could be minimized to 01 (one), since the interstitial fluid could be displaced from any point to any other point in the system without hindering the confinement of $\mathrm{OB}$ and $\mathrm{OC}$ cells, contrary to what occurs in $2 \mathrm{D}$ models.

Because of the $\mathrm{OC}$ and $\mathrm{OB}$ cell densities as well as the network topology, a value of $p_{0}=0.001$ was chosen, which corresponds to the order of magnitude established by Weinkamer [29]. The magnitude $\rho=50$ was stipulated in order to ensure rapid attraction between $\mathrm{OB}$ and $\mathrm{DS}$ up to a certain distance. Therefore, for $d=1$, the energy $E_{S}$ between an $\mathrm{OB}$ cell in direct contact with a DS (closest neighbor) takes a value of $E_{S}=\rho$. 

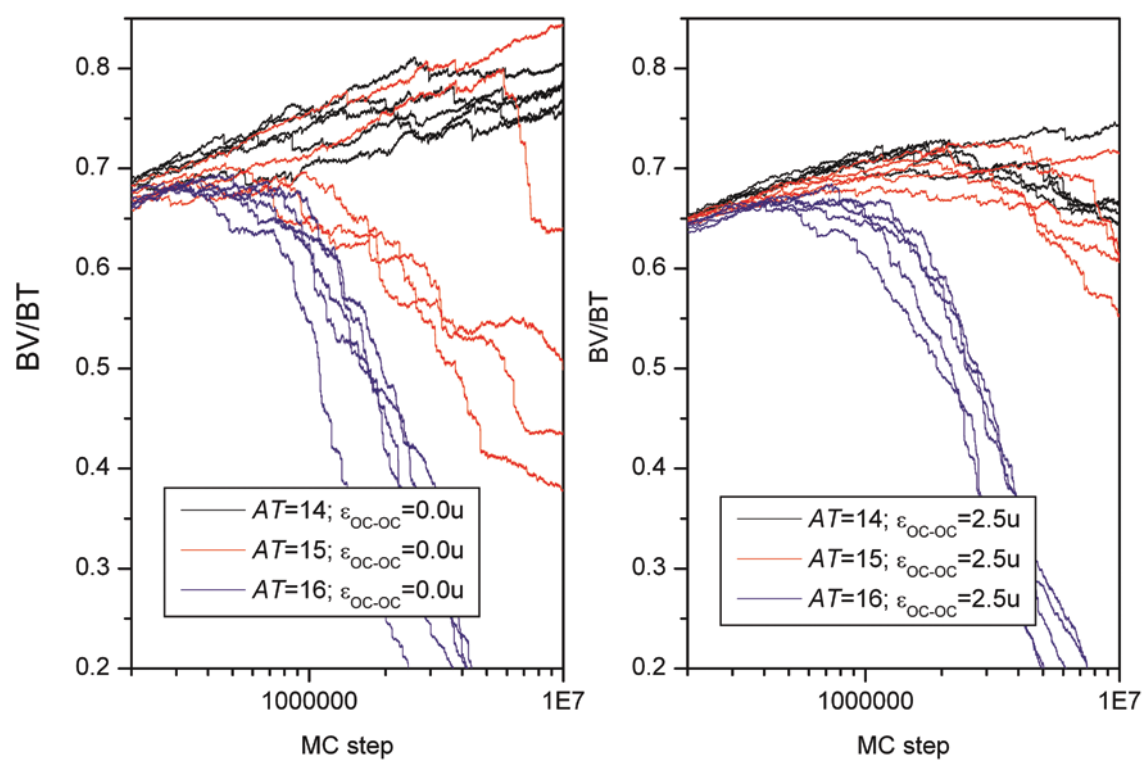

Figure 5 - Evolution of BV/BT following $10^{7}$ MCSs. Five (05) independent simulations (random initial configuration for $\mathrm{OB}$ and $\mathrm{OC}$ ) were carried out for each AT, combining (a) $\varepsilon_{O C-O C}=0.0 u$ and (b) $\varepsilon_{O C-O C}=2.5 u$. For each MCS, 120 configurations are generated. BV/BT values are calculated whenever an MCS is completed.

\section{RESULTS}

Firstly, we tried to identify the AT range for which a transition between TB loss and gain could be observed after the stipulated total number of MCSs. The behavior of the trabecular bone ratio BV/BT (number of TB cells in relation to the total number of sites in the lattice) was then accompanied for AT values of 14 , 15, and 16. For each AT value, five (05) independent simulations (different initial states for $\mathrm{OB}$ and $\mathrm{OC}$ ) were accomplished in the cases of $\varepsilon_{O C-O C}=0.0 u$ and $\varepsilon_{O C-O C}=2.5 u$ (Fig. $5 \mathrm{a}$ a-b). In both situations, we verified that transition occurs in the $14<A T<16$ range, since for $A T=14 u$ and $A T=16 u$ there is bone gain and loss, respectively, whereas the two processes take place when $A T=15 u$ (apparent instability). By comparing the differences between $\varepsilon_{O C-O C}=$ $0.0 u$ and $\varepsilon_{O C-O C}=2.5 u$, it is possible to identify a larger $\mathrm{BV} / \mathrm{BT}$ gain for $\varepsilon_{O C-O C}=0.0 u$, and the differences are more evident in the case of $A T=14 u$.

By increasing the AT transition zone and carrying out at least 05 (five) times more simulations for a smaller AT range, it was possible to detect the existence of a critical AT range around $A T=15 u$. In all the cases, there is an exponential bone mass gain until a critical time $t_{c}=10^{6} \mathrm{MCS}$ is reached. Thereafter, the behavior critically depends on the AT value for the onset of bone mass loss to take place. Stable BV/BT behavior does not occur in any of the cases, although it is steadier for $A T=14.9 u$. It is noteworthy that outside the transition zone, the exponential gain observed for $\varepsilon_{O C-O C}=0.0 u$ and $\varepsilon_{O C-O C}=2.5 u$ (Fig. 5) implies different $t_{c}$ values, and $t_{c}$ is longer in the latter case.

In an attempt to understand the reason for the criticality caused by AT, we also followed the behavior of the number of Actived Cells (AC) as a function of $A T$ and its influence on the parameter BV/BT (Figs. 6-7), since resorption and deposition directly depend on $A C$ in our model. As verified in Figure 6 , the behavior of AC changes when $t_{c}$ is reached. An exponential increase in $\mathrm{AC}$ can be noted, with a brief equilibrium period around $t_{c}$. This behavior is similar for all the $A T$ values. Nevertheless, for $A T<16$, there is an inversion in the behavior of AC; a sharp reduction takes place after $t_{c}$, and it remains saturated for a longer period at $A C \sim 25$ when $\varepsilon_{O C-O C}=2.5 u$. This is more clearly seen in Figure 7 , where saturation has a significantly larger BV/BT amplitude for $\varepsilon_{O C-O C}=2.5 u$. On the other hand, when $A T=16$, the BV/BT behavior in both cases is similar, although there is a more pronounced decrease for $\varepsilon_{O C-O C}=$ $2.5 u$ when $A C>50$.

\section{DISCUSSION}

On the basis of our analyses, we suggest that there is an "intrinsic failure" in the dynamics of bone remodeling associated with the resorption and deposition process. In the long term, this failure will affect the topology of the trabecular bone through a mecha- 

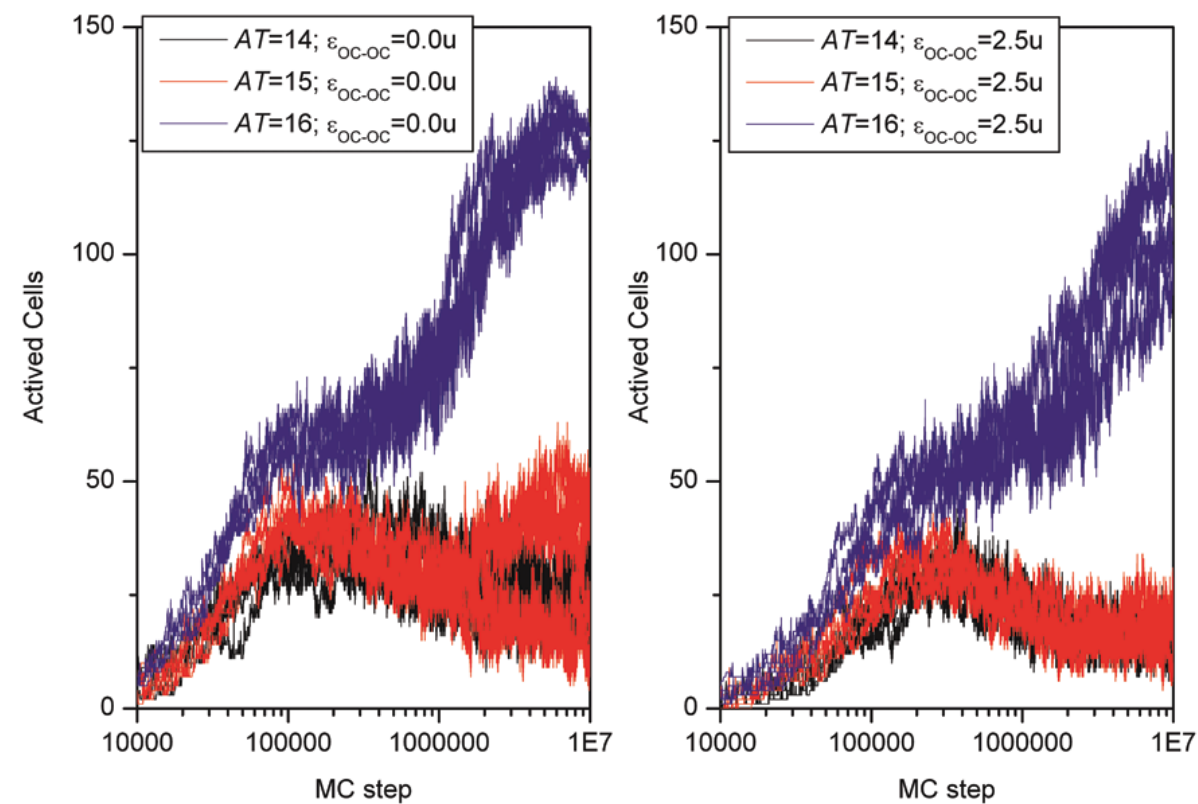

Figure $\mathbf{6}$ - Evolution of the number of cells activated by OC as a function of AT. Five (05) independent simulations are presented for each AT, shown in different colors.
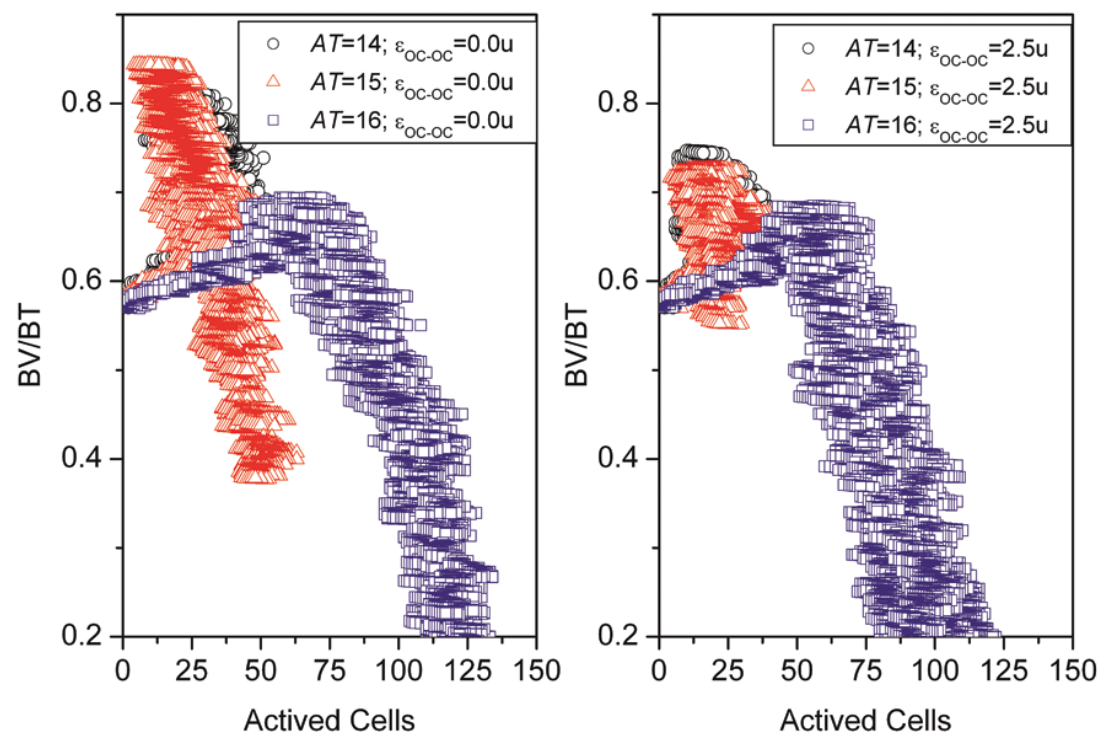

Figure $7-B V / B T$ behavior as a function of the number of cells activated by $O C$ at different ATs. Five (05) independent simulations were carried out for each AT, combining (a) $\varepsilon_{O C-O C}=0.0 u$ and (b) $\varepsilon_{O C-O C}=2.5 u$.

nism that does not depend on the balance between $\mathrm{OC}$ and $\mathrm{OB}$, causing a reduction in bone mass (Fig. 5). Such alterations become critical when the architecture of the trabecula becomes topologically unfavorable to $\mathrm{OB}$ activation. Although this hypothesis is based on our phenomenological model, it is general and valid for any other model whose $\mathrm{OC}$ and $\mathrm{OB}$ cell dynamics is explicit and occurs by means of functions that depend on the inverse of the distance. Above all, we understand that the specificity imposed by Eq. 4 and by the two conditions involved in deposition are based on first principles essential to the model. Even if the behavior of $E_{S}$ as a function of the distance were redefined $\left(1 / r, 1 / r^{2}\right.$ etc), the criticality in terms of AT observed here would still occur. This is clear from Figure 7, where it is possible to note that $E_{S}$ may affect the initial topology and trigger a new TB struc- 


\section{Case A}

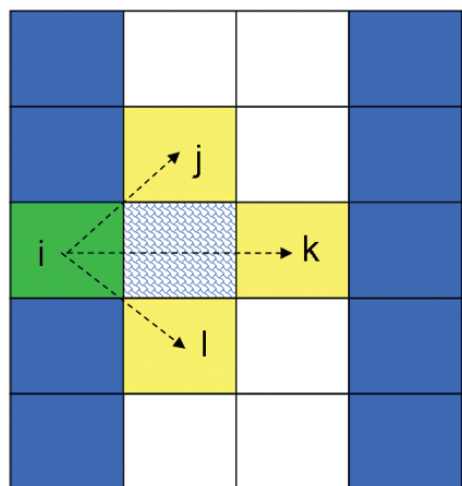

$E_{s}^{i}=E_{i j}+E_{i k}+E_{i l}$

$E_{s}^{i}=-\frac{f_{i}}{(s \sqrt{2})^{4}}-\frac{f_{i}}{(2 s)^{4}}-\frac{f_{i}}{(s \sqrt{2})^{4}}$

$E_{s}^{i}=-0.56 \frac{f_{i}}{s^{4}}$
Case B

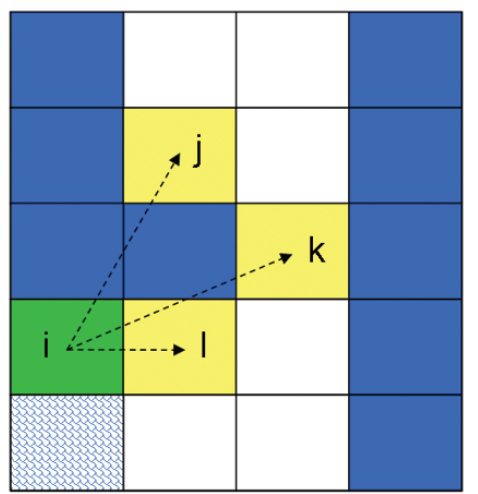

$E_{S}^{i}=E_{i j}+E_{i k}+E_{i l}$

$E_{S}^{i}=-\frac{f_{i}}{(s \sqrt{5})^{4}}-\frac{f_{i}}{(s \sqrt{5})^{4}}-\frac{f_{i}}{s^{4}}$

$E_{S}^{i}=-1.08 \frac{f_{i}}{s^{4}}$

Figure 8 - Representation of deposition by OB generating topological damage. In case A, the OB cell (green) deposits bone on the site absorbed by $\mathrm{OC}$ (shaded site), and the structure of the trabecula is maintained. Although deposition is more favored in case B because $E_{S}$ is lower than in A, deposition takes place in another site, not in the removed one.

ture that does not favor $\mathrm{OB}$ activation. It is noteworthy that the sharp decrease in BV/BT observed in Figure 7 for $A C>50$ is an evidence of this hypothesis which, in this particular case, is critical from this AC value due to the size of the network under consideration. Therefore, in an environment unfavorable to $\mathrm{OB}$ activation, the system will trigger TB resorption only, thereby resulting in acceleration of irreversible bone mass loss. Moreover, if factors associated with menopause are also considered, such as the frequency of BMU activation [30], topological failures will be amplified, leading to more precocious bone loss.

As already mentioned, $\mathrm{OC}$ cells are known as giant cells [31]. They contain various nuclei and are present in larger quantities compared with $\mathrm{OB}$ cells, which in turn are smaller and mononucleated [32]. From a physical viewpoint, resorption taking place in a certain region leaves a gap that should be later filled with $\mathrm{OB}$, thereby avoiding the loss of structural balance. Bearing in mind the size of an $\mathrm{OC}$ cell, the thickness of this gap should be much larger than the dimensions of an $\mathrm{OB}$ cell [33], thus favoring $\mathrm{OB}$ penetration and deposition. However, the thickness of this gap should decrease as it is filled with $\mathrm{OB}$, until its dimension becomes the same as that of an osteoblast [34]. This is the moment when the situation in real bones resembles the network model studied here, once the size of the site removed by $\mathrm{OC}$ is always the same as that occupied by $\mathrm{OB}$. This means that it is not possible to avoid the topological damage to the trabecular structure highlighted previously because of the different dimensions of these cells, once at some moment these dimensions will be compared. In this way, it is expected that the same type of microdamage observed in Figure 8; that is, the one caused by $E_{S}$, should also be found when cells with distinct dimensions are treated, as in the case of the real system. It is true that other microdamage could also take place along the gap, which would worsen the damaging effects on the bone structure during deposition. This reinforces the importance of the fact that $\mathrm{OC}$ cells are multinucleated, since multinucleation provides this kind of system with balance [35].

Considering $\varepsilon_{O C-O C}=2.5 u, O C$ cell clusters are generated in such a way that multinucleation is mimicked (Fig. 1, insert). Nevertheless, even though stability among the cells of this cluster exists, it does not mean that all the cells will be activated at the same time, since their activation occurs independently according to Eq. 3. Therefore, considering $\varepsilon_{O C-O C}=2.5 u$ influences "kinetics" much more than it affects the joint local action of the cells for the formation of larger cavities.

Figures 5-6 reveal there is a critical threshold for AT, which seems to be around $15 u$. When the threshold is below this critical value, bone mass loss for $\varepsilon_{O C-O C}=0.0 u$ increases 
a

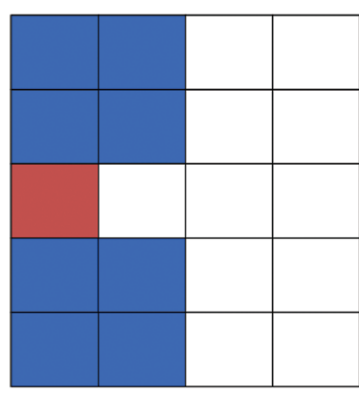

b

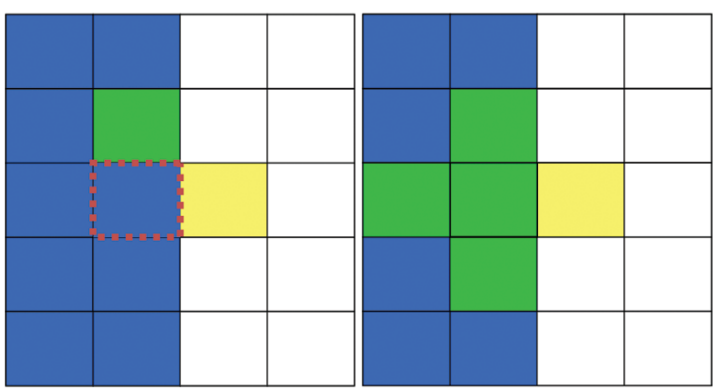

Figure 9 - Representation of the reduction in the number of activated cells (yellow) by $O C$ (red) due to failure during deposition (Fig. 10, case B). (a) Approach of OC, to remove TB (white); (b) after TB removal, only one site is activated; (c) after TB removal, the energy is not sufficient for $\mathrm{OB}$ activation, leading to the accumulation of $\mathrm{OB}$ cells (green) in the surroundings of the activated cell.

exponentially with $A T=14 u$ (Fig. 5). At a first glance, this sounds contradictory because $\mathrm{OC}$ cells have much more freedom to move and absorb TB in different parts of the surface when $\varepsilon_{O C-O C}=0.0 u$. However, a larger number of AC cells accumulate in the network compared with $\varepsilon_{O C-O C}=2.5 u$. Consequently, when $A T<15$, a higher proportion of sites involving AC cells results in DS. In this case, bone mass gain is expected to occur if the $\mathrm{OB}$ density is enough to deal with all the DS sites at the same time, thereby deactivating AC cells. On the other hand, when $A T>15$, many of the sites surrounded by AC cells do not give rise to $D S$, leading to the subsequent accumulation of $\mathrm{OB}$ cells around $\mathrm{AC}$ cells because of the ES energy. In this way, if many of these sites do not result in $E_{S}>L A$, various $\mathrm{OB}$ cells will be retained in the surroundings of $A C$ (see Fig. 9 for the representation), producing a delay in the displacement of $\mathrm{OB}$ to the other network sites (DS or not). If no deposition by OB takes place, bone mass is reduced because $\mathrm{OC}$ cells continue absorbing $\mathrm{TB}$ independent of $\mathrm{OB}$.

Metabology studies have shown that bone density rises until 30 years of age, and the bone mass peak is reached between adolescence and 35 years of age, declining thereafter due to a complex combination of factors [36]. One way to delay the onset of osteoporosis is to increase bone mass during childhood and adolescence, especially through the practice of physical activities. In the elderly, physical activity placing mechanical stress on the bone has been shown to reduce osteoporosis [37].

Nevertheless, although remodeling responds to both an increase and reduction in mechanical demand, it is easier to lose bone mass through inactivity than via a rise in mechanical stress. A bedridden young adult may have a $1 \%$ spine density loss in one week, but it will take them almost a year to regain this same mass [37]. Mechanical loads could favor a rise in $E_{S}$, thus increasing the probability of reduced bone mass loss even when $A T>15$. However, because the area covered by topological damage becomes larger with time, there would be no correspondence between resorption and deposition after a certain period, even under the action of mechanical stress capable of favoring mass gain. In other words, no matter how beneficial and efficient the strain on the bone is, this stress would be abolished in the tips because of the pores generated from deposition failure. As a consequence, the sites of the $\mathrm{AC}$ type in these regions are more susceptible to deactivation by $\mathrm{OB}$, giving rise to a more porous structure. In Figure 5, note that for $\varepsilon_{O C-O C}=2.5 u$ there is an initial bone mass gain followed by short stability in all cases. Even in the cases where the bone mass gain is larger, $A T<15$, there is inevitable onset of mass loss some time later. This suggests that even under stimulation or the action of a compound capable of altering the balance between $\mathrm{OB}$ and $\mathrm{OC}$, thereby minimizing loss, a reduction in bone mass is irreversible due to the inevitable damage to bone topology.

\section{CONCLUSION}

In this work, through a simple two-dimensional model, we reveal aspects of the kinetics of remodeling that indicate a phase transition of bone mass due to topological failures that accumulate during the deposition. Our hypothesis is that during the deposition of bone by $\mathrm{OB}$, faults can occur in the geometrical arrangement the long term and create an topological condition energetically unfavorable to $\mathrm{OB}$ activation. This condition, regardless of the activation threshold or the proportion of these cells seems to be inevitable because of the nature of the interactions that occur between $\mathrm{OB}$ and $\mathrm{TB}$. 
However, we understand that in a real system where there is presence of mechanical forces that are essential for maintaining bone mass, the effects of these failures could be minimized or delayed, but long term they still affect the loss of bone mass. This can be confirmed in our future work to include stress in our model and assess the magnitude of these flaws in a three-dimensional model. This will validate the theory here proposed in a quantitative sense. For this, more sophisticated algorithms involving finite element analysis must be implemented in our model, which will require much more cpu time to perform our exhaustive analysis.

\section{ACKNOWLEDGMENTS}

We thank Fundação Vale Paraibana de Ensino (FVE) for computational support and Fundação de Amparo à Pesquisa do Estado de São Paulo (FAPESP, Proc. 07/50894-7) for partial support of this work.

\section{REFERENCES}

[1] RODAN GA \& MATIN TJ. 1981. Role of osteoblasts in hormonal control of bone resorption - a hypothesis. Calc. Tissue Int., 33(4): 349-351.

[2] MELTON III LJ, CHRISCHILLES EA, COOPER C, LANE AW \& RIGGS BL. 1992. Perspective. How many women have osteoporosis? J. Bone Miner. Res., 7: 1005-1010.

[3] RIGGSBL \& MELTON LJ. 1986. Involutional osteoporosis. N. Engl. J. Med., 314: 1676-1686.

[4] KLEEREKOPER M, VILLANUEVA AR, STANCIU J, RAO DS \& PARFITT AM. 1985. The role of three-dimensional trabecular microstructure in the pathogenesis of vertebral compression fractures. Calcif. Tissue Int., 37: 594-597.

[5] MOSEKILDE L. 2002. Consequences of the remodelling process for vertebral. Rev. Bras. Reumatol., 42(6): 343-354.

[6] SIMONET WS, LACEY DL, DUNSTAN CR, KELLEY MSM, LUTHY R, NGUYEN HQ, WOODEN S, BENNETT L, BOONE T, SHIMAMOTO G, DEROSE M, ELLIOTT R, COLOMBERO A, TAN H, TRAIL G, SULLIVAN J, DAVY E, BUCAY N, RENSHAW-GEGG L, HUGHES TM, HILL D, PATTISON W, CAMPBELL P, SANDER S, VAN G, TARLEY J, LEE PDR \& BOYLE W. 1997. Osteoprotegerin: a novel secreted protein involved in the regulation of bone density. Cell, 309-319.

[7] LACEY D. 1998. Osteoprotegerin ligand is a cytokine that regulates osteoclast differentiation and activation. Cell, 93(2): 165175.

[8] YASUDA H. 1998. Osteoclast differentiation factor is a ligand for osteoprotegerin-osteoclastogenesis inhibitory factor and is iden- tical to TRANCE/RANKL. Proc. Natl. Acad. Sci. USA, 95: 35973602.

[9] VITOVSKI S, PHILLIPS JS, SAYERS J \& CROUCHER PI. 2007. Investigating the interaction between osteoprotegerin and receptor activator of NF-kappa B or tumor necrosis factor-related apoptosisinducing ligand. Journal of Biological Chemistry, 282(43): 31601-31609.

[10] TNAOYUKI, NOBUYUKI U \& STATSUO. 1999. A New Member of Tumor Necrosis Factor Ligand Family, ODF/OPGL/TRANCE/ RANKL, Regulates Osteoclast Differentiation and Function. Biochemical and Biophysical Research Communications, 256(3): 449-455.

[11] HSU H, LACEY DL, DUNSTAN CR, SOLOVYEV I, COLOMBERO A, TIMMS E, TAN H, ELLIOTT G, KELLEY MJ, SAROSI I, WANG L, XIA XZ, ELLIOTT R, BLACK T, SCULLY S, C CAPPARELLI SM, SHIMAMOTO G, BASS M \& BOYLE W. 1999. Tumor necrosis factor receptor family member RANK mediates osteoclast differentiation and activation induced by osteoprotegerin ligand. The National Academy of Sciences, 96(7): 3540-3545.

[12] MAGNUSSON H, LINDEN C, KARLSSON C, OBRANT KJ \& KARLSSON MK. 2001. Exercise may induce reversible low bone mass in unloaded and high bone mass in weightloaded skeletal regions. Osteoporos. Int., 12: 950-955.

[13] RALSTON SH. 1994. Analysis of gene expression in human bone biopsies by polymerase chain reaction: evidence for enhanced cytokine expression in postmenopausal osteoporosis. J. Bone Miner. Res., 9(6): 883-890.

[14] TSANGARI H, FINDLAY DM, KULIWABA JS, ATKINS GJ \& FAZZALARI NL. 2004. Increased expression of IL-6 and RANK mRNA in human trabecular bone from fragility fracture of the femural neck. Bone, 35(1): 334-342.

[15] ABDALLAH RM, STILGREN JS, NISSEN N, KASSEM M, IORGENSEN HR \& ABRAHANSEN B. 2005. Increased RANLL/OPG mRNA ratio in iliac bone biopsies from women with hip fractures. Calcif. Tissue Int., 76(2): 90-97.

[16] VON TIRPITZ C, EPP S, KLAUS J, MASON R, HAWA G \& BRINSKELLE-SCHMAL N. 2003. Effect of systemic glucocorticoid therapy on bone metabolism and the osteoprotegerin system in patients with active Crohn's disease. Eur. J. Gastroenterol. Hepatol., 15(11): 1165-70.

[17] RODRIGUEZ JI, PALACIOS J, GARCIA-ALIX A, PASTOR I \& PANIAGUA R. 1988. Effects of immobilization on fetal bone development. A morphometric study in newborns with congenital neuromuscular diseases with intrauterine onset. Calcif. Tissue Int., 43 : 335-339. 
[18] RUBIN CT \& LANYON LE. 1985. Regulation of bone mass by mechanical strain magnitude. Calcified Tissue International, 37: 411-7.

[19] ROBLING AG, CASTILLO AB \& TURNER CH. 2006. Biomechanical and molecular regulation of bone remodeling. Annu. Rev. Biomed. Eng., 8: 455-98.

[20] HERNANDEZ CJ, BEAUPRÉ GS \& CARTER DR. 2003. A theoretical analysis of the changes in basic multicellular unit activity at menopause. Bone, 32: 357-363.

[21] HING KA. 2004. Bone repair in the twenty-first century: biology, chemistry or engineering? Philos Transact A Math. Phys. Eng. Sci., 362(1825): 2821-50.

[22] OURSLER MJ, OSDOBY P, PYFFEROEN J, RIGGS BL \& SPELSBERG TC. 1991. Avian osteoclastos as estrogen target cell. Proc. Natl. Acad. Sci USA, 88: 6613-6617.

[23] HUGHES DE, DAI A, TIFFEE JC, LI HH, MUNDY GR \& BOYCE BF. 1996. Estrogen promotes apoptosis of murineosteoclasts mediated by TGF-beta. Nat. Med., 2: 1132-1136.

[24] MULVIHILL BM, MCNAMARA LM \& PRENDERGAST PJ. 2008. Loss of trabeculae by mechano-biologicalmeans may explain rapid bone loss in osteoporosis. J.R. Soc. Interface, 5: 1243-1251.

[25] RUMPLER M, WOESZ A, DUNLOP JWC, VAN DONGER JT \& FRATZL P. 2008. The effect of geometry on three-dimensional tissue growth. J.R. Soc. Interface, 5: 1173-1180.

[26] HUISKER R, RUIMERMAN R, VAN LENTHE GH \& JANSSEN JD. 2000. Effects of mechanical forces on maintenance and adaptation of form in trabecular bone. Nature, 405: 704-706.

[27] METROPOLIS N, ROSENBLUTH AW, ROSENBLUTH MN, TELLER A \& TELLER H. 1953. Equations of state calculations by fast computing machines. J. Chem. Phys., 21: 1087-1091.
[28] MANOUSIOUTHAKIS VI \& DEEM MW. 1999. Strict detailed balance is unnecessary in Monte Carlo simulation. J. Chem. Phys., 110: 2753-2756.

[29] WEINKAMER R, HARTMANN MA, BRECHET Y \& FRATZL P. 2004. A stochastic lattice model for bone remodeling and aging. Phys. Rev. Lett., 93: 2281-02.

[30] ERIKSEN EF, MOSEKILDE L \& MELSEN F. 1986. Trabecular bone remodeling and balance in primary hyperparathyroidism. Bone, (7): 213-221.

[31] FRANZOSO G, CARLSON L, XING L, POLJAK L \& SHORES EW, et al. 1997. Requirement for NF-kappaB in osteoclast and B-cell development. Genes Dev., 11: 3482-3496.

[32] DUNCAN RL \& TURNER CH. 1995. Mechanotransduction and the functional response of bone to mechanical strain. Calcified Tissue International, 57: 344-358.

[33] ERIKSEN EF \& LANGDAHL B. 1995. Bone remodeling and its consequences for bone structure. In: ODGAARD A \& WEINANS $\mathrm{H}$ (Eds.). Bone Structure and Remodeling. London, World Sci., pp. 25-36.

[34] ROBLING AG, CASTILLO AB \& TURNER CH. 2006. Biomechanical and molecular regulation of bone remodeling. Annu. Rev. Biomed. Eng., 8: 455-498.

[35] CONSENSUS DEVELOPMENT CONFERENCE. 1993. Diagnosis, prophylaxis, and treatment of osteoporosis. Am. J. Med., 94: 646-650.

[36] MOSEKILDE L. 1990. Consequences of the remodelling process for vertebral trabecular bone structure: a scanning electron microscopy study (uncoupling of unloaded structures). Bone Miner., 10(01): 13-35.

[37] NETO P \& MENDES A et al. 2002. Brazilian consensus on osteoporosis. Rev. Bras. Reumatol., 42(6): 343-354. 